\title{
Reabilitação protética de dente posterior com coroa metaloceramica e retentor intrarradicular: relato de caso
}

Prosthetic rehabilitation of back tooth with metalloceramic crown and intrarradicular seal: case report Rehabilitación protética del diente trasero con corona metallocerámica y sello intrarradicular: reporte de caso Gerbson Rodrigues de SOUZA ${ }^{1}$ José Henrique de Araújo $\mathbf{C R U Z}^{1}$ Yanna Carla Mendes SANTOS ${ }^{1}$ Itamar da Silva NUNES ${ }^{1}$ Joyce Reis CARNEIRO ${ }^{1}$ Evelinne Costa FREITAS ${ }^{1}$ Rodrigo Alves RIBEIRO ${ }^{2}$ Rodrigo Araújo RODRIGUES ${ }^{2}$

${ }^{1}$ Cirurgião(ã)-Dentista pelo Curso de Graduação em Odontologia. Centro de Saúde e Tecnologia Rural, Universidade Federal de Campina Grande, UFCG 58708-110 Patos/PB, Brasil

${ }^{2}$ Professor Doutor do Curso de Graduação em Odontologia. Centro de Saúde e Tecnologia Rural, Universidade Federal de Campina Grande, UFCG 58708-110 Patos/PB, Brasil

\section{Resumo}

A Odontologia busca soluções para a reabilitação de elementos tratados endodonticamente que normalmente se apresentam frágeis, devido a sua perca de estrutura pela cárie, e através do procedimento endodôntico, o que torna este remanescente mais frágil. Havendo assim em alguns casos a necessidade de se utilizar pinos intrarradiculares, e nesse caso os pinos préfabricados ganham espaço pelo seu uso fácil e rápido, baixo custo e não necessidade de confecção em laboratório, possibilitando o reforço a estrutura dental e promovendo uma ideal reabilitação. A paciente apresentava um elemento dental com tratamento endodôntico que após fratura da restauração coronária se apresentava sem estrutura, relatando insegurança e insatisfação com a estética dentária e funcionalidade. $O$ objetivo deste trabalho foi descrever o procedimento reabilitador em prótese dentária de uma coroa metalocerâmica sobre retentor, pino de fibra de vidro, em um elemento de número 14, destacando os materiais utilizados e a técnica de confecção, desde a desobturação do canal de escolha para inserção do pino, confecção do núcleo em resina composta até a instalação da coroa metalocerâmica. No caso clinico descrito o uso do pino de fibra de vidro se apresenta como um material de maior resistência e uma retenção ideal no interior do canal, através da sua excelente fixação com cimentos resinosos. A utilização de retentores intrarradiculares se torna essencial no tratamento reabilitador em elementos cujo seu remanescente está comprometido pela perca de estrutura dental.

Descritores: Técnica para Retentor Intrarradicular; Prótese Dentária; Estética Dentária.

\section{Abstract}

Dentistry seeks solutions for the rehabilitation of endodontically treated elements that are usually fragile, due to their loss of structure through caries, and through the endodontic procedure, which makes this remnant more fragile. Thus, in some cases, the need for intraradicular pins is required, and in this case the prefabricated pins gain space for their easy and quick use, low cost and no need to make in the laboratory, making it possible to reinforce the dental structure and promoting an ideal rehabilitation. The patient presented a dental element with endodontic treatment, which after fracture of the coronary restoration presented no structure, reporting insecurity and dissatisfaction with dental aesthetics and functionality. The objective of this study was to describe the dental restorative procedure of a metal-ceramic crown on a fiberglass pin, in an element number 14, highlighting the materials used and the technique from the preparation of the channel of choice for insertion of the pin, making the core in composite resin until the installation of the metal-ceramic crown. In the clinical case described, the use of the fiberglass pin presents itself as a material of greater strength and an ideal retention in the interior of the channel, through its excellent fixation with resin cements. The use of intraradicular retainers becomes essential in the rehabilitative treatment in elements whose remnant is compromised by the loss of dental structure.

Descriptors: Post and Core Technique; Dental Prosthesis; Esthetics, Dental.

\section{Resumen}

La odontología busca soluciones para la rehabilitación de elementos tratados endodónticamente que suelen ser frágiles, debido a su pérdida de estructura debido a la caries, y a través del procedimiento endodóntico, lo que hace que este remanente sea más frágil. Por lo tanto, en algunos casos existe la necesidad de usar clavos intraradiculares, en cuyo caso los clavos prefabricados ganan espacio debido a su uso fácil y rápido, bajo costo y sin necesidad de preparación de laboratorio, lo que permite el fortalecimiento de la estructura dental y promueve un ideal rehabilitación El paciente tenía un elemento dental con tratamiento endodóntico que, después de fracturar la restauración coronaria, no tenía estructura, informando inseguridad e insatisfacción con la estética y la funcionalidad dental. El objetivo de este trabajo fue describir el procedimiento de rehabilitación en prótesis dental de una corona de metal-cerámica en un retenedor, pasador de fibra de vidrio, en un elemento número 14, destacando los materiales utilizados y la técnica de confección, desde el desbloqueo del canal de elección para inserción del perno, haciendo el núcleo en resina compuesta hasta la instalación de la corona de metal-cerámica. En el caso clínico descrito, el uso del pasador de fibra de vidrio se presenta como un material de mayor resistencia y una retención ideal dentro del canal, a través de su excelente fijación con cementos de resina. El uso de retenedores intraradiculares se vuelve esencial en el tratamiento de rehabilitación en elementos cuyo resto se ve comprometido por la pérdida de la estructura dental.

Descriptores: Técnica de Perno Muñón; Prótesis Dental; Estética Dental.

INTRODUÇÃO

A reabilitação da estética e função dos elementos dentários tratados endodonticamente com grande perda de estrutura coronária exige em muitas das situações a utilização de pinos intracanais como forma adicional de retenção e estabilidade do material restaurador. Por sua vez o pino não tem o propósito de reforçar a estrutura do remanescente dental, e sim, promover a estabilidade e retenção aos materiais restauradores ${ }^{1}$.

Logo quando se faz referencia a dentes com tratamento endodôntico realizado, a questão da menor resistência mecânica desses elementos é logo pensada, se há possibilidade 
e/ou necessidade de reforço nesses elementos antes dos processos restauradores ${ }^{2}$.

Com essa necessidade de reforço aos elementos de menor resistência, foram introduzidos na odontologia restauradora, os pinos de fibra de vidro,como uma alternativa para substituir os pinos metálicos ${ }^{3}$. Investigações revelaram que pinos onde seu módulo de elasticidade é muito superior ao módulo da dentina, estes não reforçariam a estrutura do elemento dental, e sim transmitiriam toda a energia para a dentina fazendo gerar uma concentração de tensões maior ${ }^{4}$.

Quanto às restaurações estéticas a busca por um material que melhor mimetizem os dentes naturais está em constante aumento, sendo as cerâmicas consideradas os melhores materiais para reproduzirem as características de dentina e esmalte do elemento ${ }^{5}$.

Apesar de suas vantagens com ótimas características estéticas, as cerâmicas se apresentam com características friáveis, o que leva o desenvolvimento de uma infraestrutura metálica, uma opção que possa oferecer resistência à fratura de forma adequada a esses materiais. Com a união entre metal e cerâmica viabilizada, as restaurações metalocerâmicas, passaram a ser utilizadas amplamente ao longo dos anos com um alto nível clinico de sucesso ${ }^{6}$.

O objetivo deste trabalho foi descrever a confecção de uma prótese fixa, com coroa metalocerâmica associada a retentor intrarradicular do tipo pré-fabricado (fibra de vidro), para reestabelecimento das funções mastigatórias, estética e fonética de uma paciente na região superior.

\section{CASO CLÍNICO}

Paciente M. T. S., gênero feminino, procurou a Clínica Escola de Odontologia da Universidade Federal de Campina Grande (UFCG) apresentando um elemento dental número 14 que havia passado por tratamento endodôntico queixando-se de fratura, insegurança quanto a estética e dificuldade ao se alimentar. Foi feita anamnese e exame clínico para verificar o remanescente dental, a qualidade do tratamento endodôntico e o periápice (Figuras 1 e 2). A paciente então recebeu as instruções sobre os riscos, benefícios e a necessidade de um tratamento reabilitador, sendo então proposto para o caso a inserção de um pino de fibra de vidro e núcleo de preenchimento com coroa metalocerâmica. Após o planejamento e a seleção do pino de fibra de vidro, foi realizado o acesso ao canal com uma broca esférica número 1013 (KG
Sorensen, Cotia-SP, Brasil), o canal vestibular foi selecionado para receber o pino de fibra de vidro (WhitePost ${ }^{\circledR}$ FGM, Joinvile-SC, BRASIL), visto que os dois canais apresentavam semelhanças entre o seu tamanho e diâmetro. Prosseguindo com a desobturação do canal, as brocas Gates Glidden (Dentsply Maillefer) de números 4 e 3 foram utilizadas e posteriormente as brocas de Largo (Angelus Produtos Odontológicos S/A, Londrina-PR, Brasil) de números 3 e 2, preservando cerca de $4 \mathrm{~mm}$ do material obturador no ápice dental, limpeza da porção desobturada com hipoclorito de sódio $2,5 \%$, foi realizado a prova do pino e posterior radiografia periapical analisando a adaptação e localização do mesmo ao canal (Figura 3 e 4).

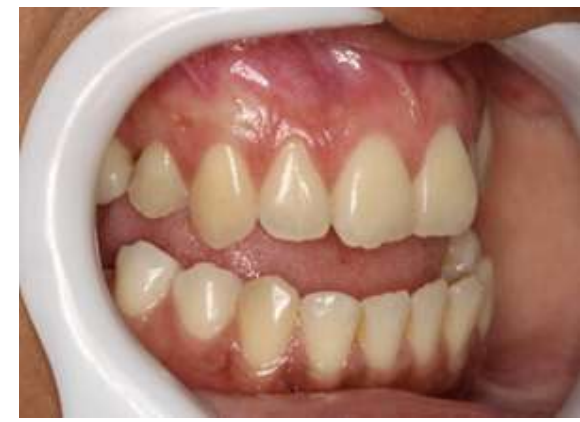

Figura 1. Aspecto inicial do elemento 14 (vista lateral).

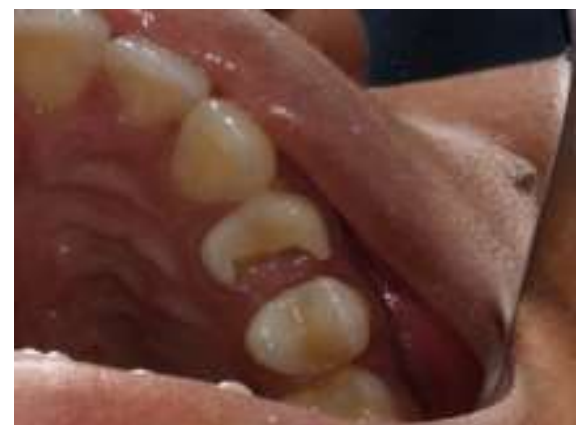

Figura 2. Aspecto inicial do elemento 14 (vista oclusal).

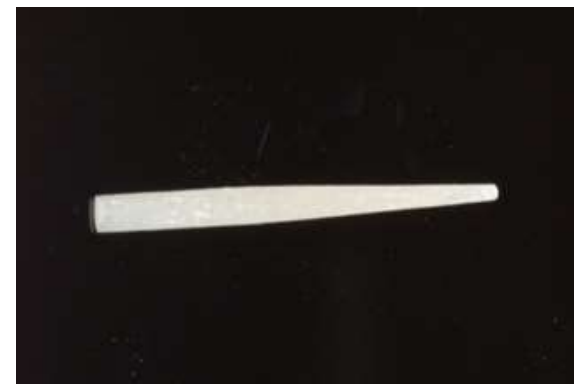

Figura 3. Pino de fibra de vidro WhitePost ${ }^{\circledR}$.

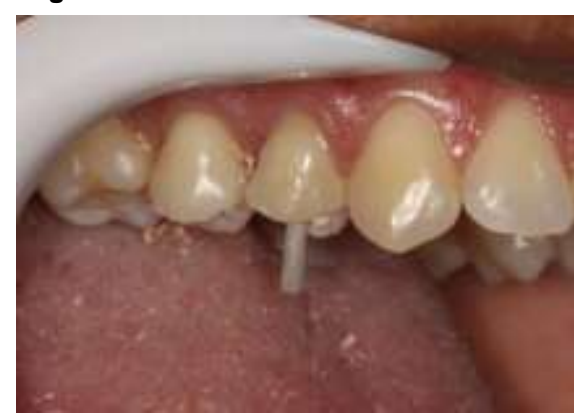

Figura 4. Pino adaptado no canal radicular. 
Em seguida, cimentou-se o pino de fibra de vidro, obedecendo 0 protocolo de condicionamento ácido com gel fosfórico a 37\% (Maquira Indústria de Produtos Odontológicos $\mathrm{S} / \mathrm{A}$, Maringá-PR, Brasil), durante 15 segundos intracanal e 40 segundos no pino, após esse tempo foi realizada a lavagem com água destilada pelo dobro de tempo, intracanal e no pino. Foi realizada a secagem do canal com cones de papeis absorventes endodônticos (Dentsply Maillefer), o protocolo seguiu com a aplicação do Silano (Maquira Indústria de Produtos Odontológicos S/A, Maringá-PR, Brasil) no pino, aguardando cerca de 1 minuto para evaporação, em seguida a aplicação do sistema adesivo Master Bond DE (Biodinâmica, Ibiporã-PR, Brasil) com auxílio de microbrush (KG Sorensen, Cotia-SP, Brasil) no canal e no pino em duas camadas seguidas por fotopolimerização de 20 segundos. Posteriormente, aplicou-se o cimento resinoso Allcem Core (FGM, Joinvile-SC, Brasil) no canal, sendo inserido em movimento único até seu extravasamento, com finalidade de diminuir possíveis bolhas de ar, seguido pela inserção do pino no canal e estabilização, enquanto fotopolimerizou-se por 40 segundos. Imediatamente foi realizada a confecção do núcleo de preenchimento com resina composta ESPE Z100 cor UD (3M, Sumaré-SP, Brasil) pela técnica incremental de $2 \mathrm{~mm}$ (Figura 5 e 6 ).

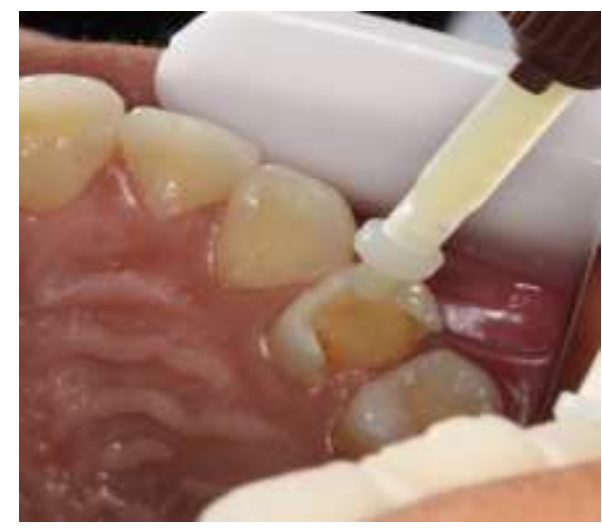

Figura 5. Aplicação do cimento resinoso no canal radicular.

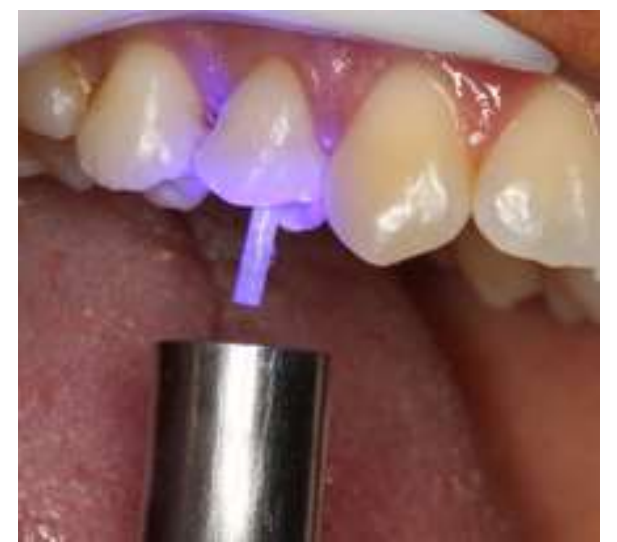

Figura 6. Fotopolimerização do pino de fibra de vidro.
O procedimento continuou com o corte do pino de fibra de vidro com o auxílio de uma broca tronco cônica de número 2135 (KG Sorensen, Cotia-SP, Brasil), sendo feitos ajustes oclusais com a broca do tipo chama de vela número 3118 (KG Sorensen, Cotia-SP, Brasil). Em uma segunda seção foi realizada a confecção do preparo do elemento dental para receber a coroa metalocerâmica, com uma broca esférica de número 1013 (KG Sorensen, Cotia-SP, Brasil) em inclinação de $45^{\circ}$ em relação ao longo eixo do dente, na altura cervical foram feitas canaletas na face vestibular e palatina do elemento para orientação, após com broca tronco cônica 2135, foram confeccionadas sulcos nas faces vestibular e palatina no sentido do longo eixo do dente, com a mesma broca as canaletas foram unidas dando uma forma de conveniência ao preparo, em seguida com a broca 3195FF (KG Sorensen, Cotia-SP, Brasil) foram rompidos os pontos de contatos mesial e distal (Figuras 7).

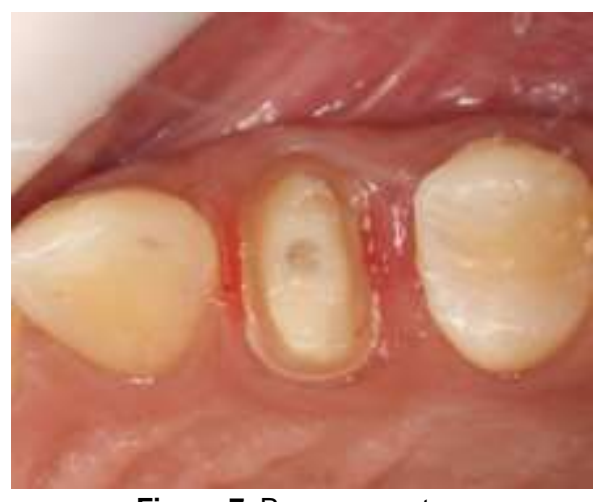

Figura 7. Preparo pronto.

Após a confecção do preparo foi realizada a moldagem da arcada superior, primeiramente foram inseridos fios retratores previamente mergulhados na solução Hemopare (Maquira Indústria De Produtos Odontológicos S/A, Maringá-PR, Brasil), buscando auxiliar na hemostasia e retração gengival. Se inseriu o fio de diâmetro menor 000-Retraflex (Biodinâmica, Ibiporã-PR, Brasil), em seguida o fio mais calibroso 00-Retraflex (Biodinâmica, Ibiporã-PR, Brasil).

Após a inserção dos fios retratores gengivais, com moldeira de estoque perfurada (Tecnodent Indústria E Comércio, IndaiatubaSP, Brasil) previamente selecionada por tentativa, o silicone de adição pasta denso/pesada (DFL Indústria e Comércio S.A., Rio De Janeiro-RJ, Brasil) foi preparado e inserido, com pressão vigorosa para bom escoamento do material e boa marcação na moldagem da arcada. Logo após ser retirado da cavidade, o fio retrator de diâmetro mais calibroso foi removido, em seguida dentro do 
molde e na região do elemento foi inserido o silicone de adição fluido/leve (DFL INDÚSTRIA E COMÉRCIO S.A., RIO DE JANEIRO-RJ, BRASIL), sendo então a moldeira novamente levada à boca que após o tempo de presa a moldeira foi retirada da arcada e o fio retrator de menor diâmetro foi removido do sulco gengival. Para a moldagem da arcada antagonista, foi selecionada uma moldeira de estoque perfurada e 0 uso de silicone de adição pasta denso/pesada (Figuras 8).

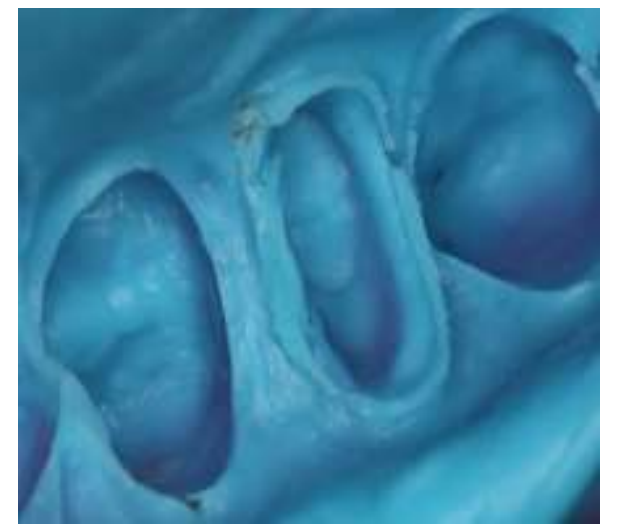

Figura 8. Molde aproximado do preparo do elemento 14.

Foi confeccionada uma coroa provisória com o uso de um elemento de estoque (Dentsply Indústria e Comércio Ltda, PetrópolisRJ, Brasil), devidamente desgastado ate sua adaptação à margem cervical, o preparo foi devidamente incrustado com vaselina sólida (Farmax, Divinópolis-MG, Brasil), foi preparada uma porção de resina acrílica (Vipiflash-Vipi), que foi inserida na porção palatina do preparo, unindo o dente de estoque, durante seu tempo de presa, por algumas vezes foi retirada e colocada a coroa provisória, visando a sua anatomização no remanescente, após seu tempo de presa, a coroa provisória foi retirada e desgastada, buscando a sua melhor adaptação marginal e oclusal. A coroa provisória foi então cimentada com cimento a base de óxido de zinco sem eugenol PROVY (Dentsply Indústria e Comércio Ltda, Petrópolis-RJ, Brasil).

Em uma terceira sessão, foi realizada a prova do coping metálico, analisando a sua estrutura e retenção, e moldagem de transferência com alginato Algi-Gel (Maquira Indústria De Produtos Odontológicos $S / A$, Maringá-PR, Brasil). Logo após a retirada do molde foi inserido no interior do coping resina acrílica Dencrilay (Dencril Produtos Odontológicos, Pirassununga-SP, Brasil), e foi estabilizado um pino de latão PREVEN (Preven Ind. e Com. de Produtos Odontológicos Eireli Epp, Guapirama-PR, Brasil), confeccionando assim o troquel de núcleo do elemento, foi realizado vazamento do molde em gesso especial do tipo IV DURONE (Dentsply Indústria e Comércio Ltda, Petrópolis-RJ, Brasil), que em seguida foi encaminhado para o laboratório protético para confecção da cerâmica (Figuras 9 e 10).

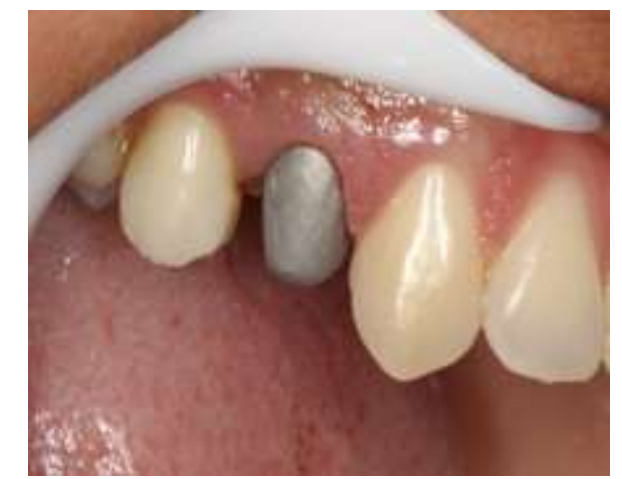

Figura 9. Coping metálico adaptado ao preparo dental.

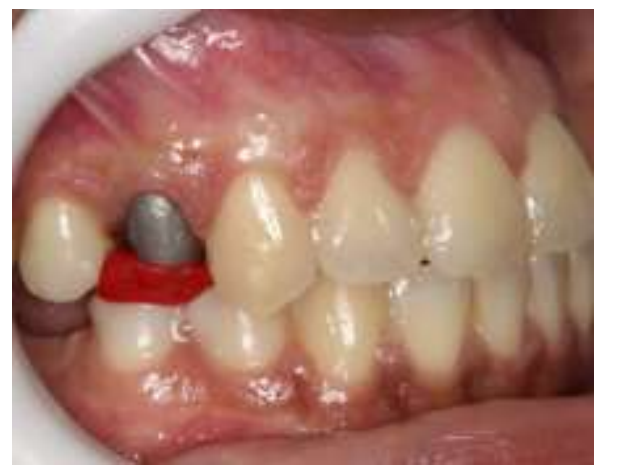

Figura 10. Registro de oclusão com coping metálico.

$\mathrm{Na}$ quarta sessão foi feita a prova da peça ao dente observando a sua excelente adaptação, além disso, o material utilizado para a cimentação foi o cimento resinoso Allcem Core (FGM, Joinvile-SC, Brasil) (Figura 11).

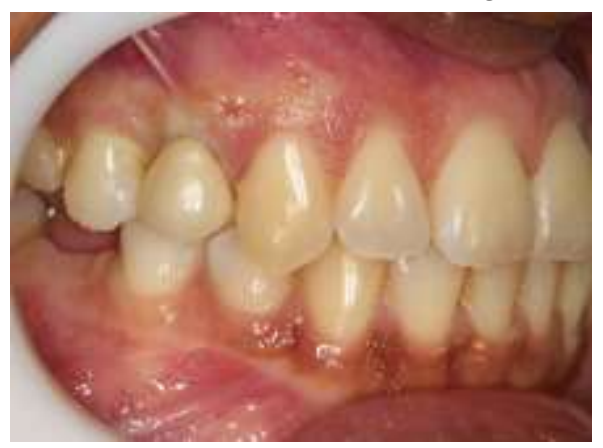

Figura 11. Coroa metalocerâmica cimentada.

Os ajustes oclusais foram realizados e a paciente recebeu instruções quanto à higienização e controles pós-instalação.

DISCUSSÃO

Estudos mostram dentes com raízes fragilizadas pelo tratamento endodôntico, os pinos de fibra de vidro são eficientes retentores, se sobressaindo, sobre o preparo para colocação dos retentores intrarradiculares ou por restaurações anteriores metálicas que perdem a sua retenção ${ }^{7}$.

Em outros estudos, ao optar por um 
retentor radicular, sendo ele o pino de fribra de vidro, alguns fatores devem ser analisados, como: a quantidade de tecido coronário remanescente, tamanho e conformidade do canal radicular, oclusão e posição do elemento. O profissional deve estar atento as diferenças existentes entre os tipos de pinos intracanais, podendo assim selecionar aquele mais apropriado para cada caso e situação ${ }^{8}$.

Em dentes posteriores com tratamento endodôntico as reabilitações além de restabelecerem a função, tem como papel fornecer resistência perante as cargas oclusais, pois esses elementos são geralmente mais acometidos a fraturas do que dentes vitais, devido a sua perda de tecido de suporte ${ }^{9}$. Para outros autores, a longevidade de dentes com tratamento endodôntico está diretamente ligada ao remanescente dentário e à eficácia dos procedimentos restauradores, mostrando assim que tanto em procedimentos endodônticos, quanto restauradores, há importância de se optar por procedimentos conservadores ${ }^{10}$.

Autores avaliaram em um estudo com pinos de fibra de vidro pré-fabricados e pinos de aço inoxidável, que após seis meses de suas colocações a taxa de sobrevivência dos pinos em fibra de vidro foi consideravelmente maior que os pinos de aço inoxidável ${ }^{11}$.

Quanto a cimentação desses retentores intrarradiculares autores relataram uma diversidade de materiais que já foram avaliados e propostos, porém, os que têm eleição são os cimentos resinosos pois apresentam propriedades físicas e biológicas que auxiliam na escolha do agente de cimentação, levando em conta características como a manipulação do cimento em associação a partículas do material restaurador ${ }^{12}$. Neste contexto autores, propuseram que os cimentos resinosos trazem uma melhor proporção de selamento marginal, propriedades físicas e capacidade de retenção que se mostra superior quando comparado aos cimentos a base de água ${ }^{13}$.

A cerâmica dental vem a ser na odontologia restauradora um dos materiais mais utilizados no cotidiano, já que se observa uma crescente demanda por materiais estéticos ${ }^{14}$. Introduzido na odontologia na década de 1950, o sistema que traz a combinação entre metal e ceramica trouxe uma significativa alteração na prática odontológica. Através da associação entre o metal e a sua resistência com a cerâmica e sua qualidade estética, tornando-se possível a realização de restaurações unitárias e múltiplas que fossem compatíveis com os tecidos orais e que restabelecesse as funções estéticas e mastigatórias ${ }^{15}$.
Para alguns autores grande parte dos profissionais enxergam na cor acinzentada da infraestrutura metálica como no caso da liga níquel - cobre das coroas metalocerâmicas, um elemento que afeta de maneira negativa a estética. No entanto se observou que o problema está na espessura da camada opaca, que deve fazer uma completa ocultação na cor do metal, uma camada de menor espessura proporcionaria uma visão de menor valor na restauração, não refletindo a luz recebida na sua superfície na mesma intensidade que os demais elementos dentais, refletindo assim a cor acinzentada ${ }^{16}$.

Por outro lado os mesmos autores afirmaram ainda que uma espessura maior pode por muitas vezes opacificar a estrutura de forma exagerada, podendo refletir a luz recebida em uma intensidade muito maior que os demais elementos dentais, o que aumentaria de forma considerável a percepção visual pela cor esbranquiçada. Sendo uma espessura de $0,2 \mathrm{~mm}$ de porcelana opaca uma quantidade ideal para se realizar o mascaramento ${ }^{16}$.

Em outros estudos autores relataram que uma camada de $0,1 \mathrm{~mm}$ não reproduz de forma confiável a cor da porcelana opaca ${ }^{17}$. Contudo, autores afirmam em um estudo longitudinal de acompanhamento clínico por 20 anos que tomando os devidos cuidados é possível ter resultados satisfatórios com coroas metalocerâmicas em relação a cor e opacidade $^{18}$.

Em seus estudos autores avaliaram 100 coroas metalocerâmicas unitárias, pertencentes a 50 pacientes, sendo destes 34 mulheres e 16 homens, onde os autores puderam constatar que $97 \%$ não apresentaram queixas sobre as coroas no item da sua estética. Analisando esses dados pode-se perceber que a habilidade do técnico em prótese em mascarar a infraestrutura metálica de forma apropriada, tem uma grande participação no resultado estético final da prótese fixa ${ }^{19}$.

O sucesso desse procedimento protético passa, portanto, pela necessidade de respeitar as normas de preparo em elementos dentais que irão receber uma coroa metalocerâmica, como o contorno gengival e a estabilidade oclusal. Alem de fazer com que o preparo se mantenha no limite intrasulcular, o que é essencial para se obter a estética, a saúde do periodonto e previsibilidade no resultado ${ }^{20}$, tendo como objetivo o restabelecimento da simetria $^{21}$, recuperando a fisiologia mastigatória, função, harmonia e estética entre os arcos dentários ${ }^{22}$. 
CONCLUSÃO

Pode-se concluir que a confecção de coroa metalocerâmica com o uso de pino de fibra de vidro em elementos com tratamento endodôntico em região de pré-molares superior responde satisfatoriamente para os restabelecimentos funcionais, estéticos e fonéticos no processo de reabilitação do paciente.

\section{REFERÊNCIAS}

1. Reis BR, Soares PBF, Castro CG, Santos Filho PCF, Soares PV, Soares CJ. Uso de coroa em cerâmica pura associada a pino de fibra de vidro na reabilitação estética do sorriso: relato de caso. ROBRAC. 2019;19(50):264-69.

2. Ferreira R, Mildemberg B, Gadotti BC, Garcia RN. Avaliação da influência do tratamento endodôntico na resistência de união de pinos de fibra reforçados por um compósito restaurador. RSBO. 2011;8(2):174-81.

3. Baldissera $P$, Zicari $F$, Valandro LF, Scotti R. Effect of root canal treatments onquartz fiberposts bonding to root dentin. J Endod. 2006;32(10):985-88.

4. Alessandro V, Simone G, Carel LG. An SEM evaluation of several adhesive systems used for bonding fiber posts under clinical conditions. Dental Mater.2002;18(7):495-502.

5. Miyashita E. Odontologia estética: planejamentoe técnica. São Paulo: Artes Médicas. 2006.

6. Borges GA, Spohr AM, Correr Sobrinho L, Consani S, Sinhoreti MAC. História e atualidade das cerâmicas odontológicas. Rev ABO Nac. 2001;9(2):112-17.

7. Tanoue $\mathrm{N}$, Nagano $\mathrm{K}$, Shiodo $\mathrm{H}$, Matsumura $\mathrm{H}$. Application of a pre-mpregnatedfiber-reinforced composite in the fabrication of an indirect dowel-core. J Oral Sci. 2007; 49(2):179-82.

8. Parčina I, Amižić, Baraba A. Esthetic Intracanal Posts. Acta Stomatol Croat. 2016;50(2):143-50.

9. Bitencourt C, Nunes MM. Avaliação clínica retrospectiva interina de restaurações diretas de resina compostas e coroas cerâmicas com pino de fibra de vidro e núcleo metálico fundido [monografia]. Porto Alegre: Universidade Federal do Rio Grande do Sul. Faculdade de Odontologia; 2017.

10. Scotti N, Forniglia A, Tempesta RM, Comba A, Saratti CM, Pasqualini $D$ et al. Effects of fiberglass-reinforced composite restorations on fracture resistance and failure mode of endodontically treated molars. J Dent. 2016;53:82-7.

11.Gbadebo OS, Ajayi DM, Oyekunle OO, Shaba PO. Randomized clinical study comparing metallic and glass fiber post in restoration of endodontically treated teeth. Indian J Dent Res. 2014;25(1):58-63.
12. Fabianelli A, Goracci C, Bertelli E, Davidson CL, Ferrari M. A clinical trial of Empress II porcelain inlays luted to vital teeth with a dualcuring adhesive system and a self-curing resin cement. J Adhes Dent. 2006;8(6):427-31.

13. Ernst CP, Cohnen U, Stender E, Willershausen $B$. In vitro retentive strength of zirconium oxide ceramic crowns using different luting agents. J Prosthet Dent. 2005;93(6):551-58.

14. Camargo AMA, Fonseca ACJM, Melo Filho AB, Balduccii, Bacigalupo JC, Salgado JAP. Crowns fit of the Procera's system in two types of cervical limits. Rev Odontol UNESP. 2004;33(3):109-14.

15. Ribeiro VAQ, Sousa RC, Paiva AEM, Vasconcelos LMR, Lima GR, Santana IL. Avaliação do desajuste marginal de copings para coroa total metalocerâmica em términos cervicais tipo chanfro e ombro com bisel 45․ RFO UPF.2010;15(3):281-85.

16. O'Connor RP, Mackert JR Jr, Myers ML, Parry EE. Castability, opaque masking, and porcelain bonding of 17 porcelain-fused-to-metal alloys. J Prosthet Dent. 1996;75(4):367-74.

17. Ozcelik TB, Yilmaz B, Ozcan I, Kircelli C. Colorimetric analysis of opaque porcelain fired to different base metal alloys used in metal ceramic restorations. J Prosthet Dent. 2008;99(3):193-202.

18. Näpänkangas $R$, Raustia A. Twenty-year follow-up of metal-ceramic single crowns: a retrospective study. Int $\mathrm{J}$ Prosthodont. 2008;21(4):307-11.

19. Hoppen LGC, Garbin CA, Rigo L, Schuh C, Federizzi L. Comparação estética entre coroas confeccionadas com os sistemas cubo e metalocerâmico. RSBO. 2010;7(2):146-53.

20. Chu FC, Frankel N, Smales RJ. Surface roughness and flexural strength of self-glazed, polished, and reglazed In-Ceram/Vitadur Alpha porcelain laminates. Int $\mathrm{J}$ Prosthodont. 2000;13(1):66-71.

21. Silveira D, Campos CDA, Souto VK, Xavier IS, Junior ESB, Dias VO. Reanatomização de incisivo lateral conoide em odontopediatria: relato de caso. Rev Intercâmbio.2017;8:260-70.

22. Sá TM, Cruz JHA, Guênes GMT, Almeida MSC, Medeiros LADM, Figueiredo CHMC. A importância da anatomia e escultura dental para prática de procedimentos clínicos odontológicos. Arch Health Invest 2018;7 (Spec Iss 7):6. 


\section{CONFLITO DE INTERESSES}

Os autores declaram não haver conflitos de interesse

\section{AUTOR PARA CORRESPONDENCIA}

José Henrique de Araújo Cruz

Rua Paulo Diogenes, 57 - Centro

59990-000 Rafael Fernandes - RN, Brasil

Telefone: (83) 99625-0125.

E-mail: henrique_araujo1992@hotmail.com

Submetido em 04/04/2020

Aceito em 23/10/2020 